\title{
Il Senso della Contumacia
}

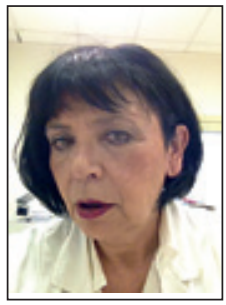

Chiara Cherubini

\section{Caro Marco,}

Varie organizzazioni internazionali hanno prodotto Linee Guida e raccomandazioni per l'implementazione nel "setting emodialitico" sulla prevenzione e sul controllo delle infezioni: Centers for Disease Control and Prevention (CDC), Association of Professional in Infection Control (APIC), Kidney Disease Outcomes Quality Initiative (K/DOQI), European Best Practice Guidelines/European Renal Best Practice (EBPG/ERBP), Kidney Disease: Improving Global Outcomes (KDIGO). Trovo auspicabile e necessario, alla luce delle nuove conoscenze, stendere Linee Guida nazionali che garantiscano ai pazienti comportamenti univoci e che orientino le organizzazioni sanitarie e allocare le risorse economiche, più che ad una contumacia "demone della paura", oltremodo costosa e non garantista, al recupero della cultura della qualificazione e della preparazione delle risorse umane, con la reale disponibilità di imprescindibili sussidi operativi, fatto che, oserei dire, oggi potrebbe portare alla bonifica di alcune problematiche infettive e garantire a tutti i pazienti infetti e non, la possibilità di non contrarre ulteriori patologie emergenti.

Come reviewer del tuo lavoro, "Il punto su epatite B e C in dialisi: riflessioni sulla contumacia dei pazienti", sento di dover fare il seguente breve commento.

Nella mia città sono in atto cambiamenti importanti, tra cui lo smembramento recente di unità operative con dispersione di pazienti dializzati in strutture private accreditate, fatta eccezione per alcuni rientri per gravi condizioni cliniche. Questo sarebbe di buon auspicio, se lo si vede dal punto di vista dell'acquisizione del fatto "della non necessità di un Centro dedicato alle problematiche infettive". I pazienti, possono essere seguiti ovunque... se assistiti con le dovute attenzioni/precauzioni!

Comunque, il problema della trasmissione ospedaliera delle patologie infettive in genere, cosa di non poco conto, rimane, a mio avviso, un problema aperto.

A tale proposito, nella Regione Lazio, è stato redatto un Decreto Commissariale n. U0090/2010 (20/11/2010 - supplemento ordinario n. 197 al Bollettino Ufficiale n. 43) per la regolamentazione dei Centri Dialisi.

Al punto 3.5.1.1 dell'allegato 1 si prevede espressamente che, nei Centri Dialisi ambulatoriali:

... "devono essere presenti: una sala dialisi contumaciale per pazienti infetti (con almeno un posto dialisi dedicato) dotata di spogliatoi, servizi igienici con interposta zona filtro...";

... "devono essere garantiti monitor dedicati per pazienti HBV, HCV, HIV positivi e per dialisi in emergenza”.

La questione è stata oggetto di vivaci discussioni in sede di Commissione di vigilanza dell'emodialisi nel Lazio, dove io sono stata anche chiamata a far parte come consulente. Non mi sembra che l'esperienza maturata allo Spallanzani sia ser- vita o che, almeno, sia stata utilizzata se, con la buona pace e soddisfazione della Commissione di vigilanza, è stata redatta, in data 9 Marzo 2012, una nota esplicativa, da parte della Regione al suddetto Decreto, con particolare riferimento alla modalità di utilizzo della "sala contumaciale e del posto dialisi in essa previsto", nonché "delle apparecchiature da dedicare ai pazienti infetti", che recita:

... ferma restando la presenza di "una sala dialisi contumaciale per pazienti infetti (con almeno un posto dialisi dedicato) dotata di spogliatoi, servizi igienici con interposta zona filtro..." nella quale "l'assistenza al paziente in trattamento dialitico... deve essere effettuata da personale infermieristico specificatamente dedicato", essa si intende dedicata a pazienti portatori di HBsAg o $\underline{\text { di altre }}$ patologie infettive con rischio di contagio in atto.

Tale sala sarà utilizzata per i dializzati portatori di infezioni in fase di attività e in relazione alle necessità cliniche di isolamento secondo procedure predefinite dal responsabile sanitario del Centro Dialisi; in assenza di patologie infettive, essa potrà essere utilizzata in accordo con le esigenze della struttura.

Corre, comunque, 1'obbligo per il responsabile sanitario del Centro di adottare un'organizzazione del servizio tale da poter accogliere immediatamente eventuali richieste di trattamento dialitico da parte dei pazienti con insufficienza renale acuta o cronica portatori di HBsAg o di altre patologie infettive, come prima specificato. Il responsabile sanitario della struttura dovrà garantire ai predetti pazienti la terapia dialitica nel posto a essi riservato nella sala contumaciale, non consentendo a pazienti non protetti da anticorpi contro il virus B di essere trattati, anche in altri turni, nei posti dialisi attigui.

Infine, il mio commento esorta tutti gli addetti ai lavori a considerare sia nel senso divulgativo che, soprattutto, in quello scientifico la necessità di praticare studi adeguati sulla possibilità del trattamento terapeutico di questi pazienti e sull'importanza del coinvolgimento di epatologi e/o infettivologi e/o gastroenterologi nelle problematiche renali degli stessi: si può affermare che ormai esiste la possibilità di trattare tutti. A breve, dando giustamente credito alle parole del ministro della salute Lorenzin, anche l'epatite $\mathrm{C}$ potrà essere debellata! Per l'epatite B, l'Adefovir e il Tenofovir sono farmaci ben tollerati e quest'ultimo non ha dato resistenze!

Che devo dire di più? Ai posteri l'ardua sentenza.

Chiara Cherubini

Indirizzo dell'Autore:

Dr.ssa Chiara Cherubini

U.O.S. Nefrologia-Dialisi nelle Patologie Infettive

A.O. S. Camillo Forlanini

Via Portuense 292

00149 Roma

ccherubini@scamilloforlanini.rm.it

\footnotetext{
Accettato: 27 Maggio 2014
} 